\title{
Outcomes of salvage liver transplant for recurrent hepatocellular carcinoma: A comparison with primary liver transplant
}

\author{
Yuxin Guo1, Ek-Khoon Tan ${ }^{1}$, Thinesh-Lee Krishnamoorthy², Chee-Kiat Tan ${ }^{2}$, Ban-Hock Tan ${ }^{3}$, \\ Thuan-Tong Tan $^{3}$, Ser-Yee Lee ${ }^{1}$, Chung-Yip Chan ${ }^{1}$, Peng-Chung Cheow ${ }^{1}$, \\ Alexander Y. F. Chung ${ }^{1}$, Prema Raj Jeyaraj ${ }^{1}$, and Brian K. P. Goh ${ }^{1,4}$ \\ Departments of ${ }^{1}$ Hepatopancreatobiliary and Transplant Surgery, ${ }^{2}$ Gastroenterology and Hepatology, and \\ ${ }^{3}$ Infectious Disease, Singapore General Hospital, ${ }^{4}$ Duke-NUS Medical School, Singapore
}

\begin{abstract}
Backgrounds/Aims: Salvage liver transplantation (SLT) is a therapeutic strategy for recurrent hepatocellular carcinoma (HCC). However, it remains controversial with compromised survival outcomes and increased perioperative morbidity compared to primary liver transplant (PLT). In the present work, we describe our institution's experience on SLT by comparing outcomes of SLT to PLT for HCCs. Methods: Retrospective analysis was conducted for 49 transplant patients from 2006-2017. A comparative analysis was carried out between 14 SLT patients and 35 PLT patients. Results: SLT patients demonstrated significantly shorter time to recurrence than PLT patients (median=5.5 versus 23 months, $p<0.001)$ with a trend towards increased perioperative major morbidity $(42.9 \%$ versus $37 \%, p=0.711)$, inferior 5 -year overall survival $(61 \%$ versus $75 \%, p=0.345)$ and inferior 5 -year recurrence-free survival $(57 \%$ versus $72 \%, p=0.263)$. However, overall survival from the point of primary resection over a 10-year period showed no statistical difference between the 2 groups (SLT=60\% versus PLT=61\%, $p=0.685$ ). Conclusions: $S L T$ is a viable treatment strategy for HCCs. However, it exhibited poorer short-term perioperative and oncologic outcomes than PLT. SLT requires better patient selection with liver donor grafts for optimization of resource allocation in this era of organ shortage. Considering the worldwide shortages in liver grafts, it is hypothesized that optimization of a salvage transplant strategy may improve resource allocation and reap optimal patient outcomes. (Ann Hepatobiliary Pancreat Surg 2019;23:1-7)
\end{abstract}

Key Words: Hepatocellular carcinoma; Salvage liver transplantation; Primary liver transplantation; Survival

\section{INTRODUCTION}

Hepatocellular carcinoma (HCC) remains a leading cause of death from cancer worldwide. ${ }^{1}$ While hepatic resection and liver transplantation are curative options, the most optimal treatment modality remains debatable. Liver resection is hampered by high recurrence rates of up to $76 \%{ }^{2,3}$ Though primary liver transplantation (PLT) for small resectable HCCs shows favourable survival and disease-free outcomes, its utility is restricted by the limited availability of liver grafts. ${ }^{4,5}$

In view of worldwide organ shortage, a salvage liver transplant (SLT) strategy was proposed earlier. For an SLT, hepatic resection is performed as a first-line treatment for HCC and liver transplantation is reserved in the event of tumour recurrence or deterioration in liver function., ${ }^{2,5}$ However, several concerns were raised regarding a salvage strategy. Firstly, SLT may be associated with higher perioperative morbidity and mortality due to the technical complexities as a result of dense adhesions and portal hypertension in patients. ${ }^{6-9}$ Secondly, long-term oncological outcomes of SLT for recurrent HCC may be poorer compared to PLT, which potentially leads to organ wastage. 9 Finally, potential SLT and PLT patients run the risk of disease progression beyond criteria while waiting for an available organ. ${ }^{3,5}$ Hence, due to limited graft availability, repeat liver resection (RLR) has been widely adopted as a viable alternative to SLT for recurrent HCC. ${ }^{10,11}$ However, the feasibility of RLR for recurrent HCC is limited by the adequacy of future liver remnant and liver function. RLR is

Received: June 13, 2018; Revised: October 6, 2018; Accepted: October 7, 2018

Corresponding author: Brian K. P. Goh

Department of Hepatopancreatobiliary and Transplant Surgery, Singapore General Hospital, 20 College Road, 169608, Singapore Tel: +65-63265564, Fax: +65-62209323, E-mail: bsgkp@hotmail.com

Copyright (C) 2019 by The Korean Association of Hepato-Biliary-Pancreatic Surgery

This is an Open Access article distributed under the terms of the Creative Commons Attribution Non-Commercial License (http://creativecommons.org/ censes/by-nc/4.0) which permits unrestricted non-commercial use, distribution, and reproduction in any medium, provided the original work is properly cited. Annals of Hepato-Biliary-Pancreatic Surgery • pISSN: 2508-5778 - eISSN: 2508-5859 
also associated with a high risk of cancer recurrence. ${ }^{12-15}$

Several studies compared SLT as an integrated strategy to PLT with varying conclusions. Some of the studies associated SLT with poor survival outcomes and greater morbidity, ${ }^{6,16,17}$ while others supported SLT as a safe and feasible option enabling an effective resource allocation. ${ }^{17,18}$ Considering the controversies surrounding SLT, we initiated the present study to evaluate our institution's experience with patients undergoing SLT and to compare their outcomes with patients undergoing PLT for HCC.

\section{MATERIALS AND METHODS}

Between June 2007 and February 2017, 49 consecutive patients who underwent liver transplant for $\mathrm{HCC}$ at the Singapore General Hospital, were retrospectively reviewed from our prospectively maintained liver transplant database. Institutional review board approval was obtained prior to conducting the study. Fourteen patients underwent SLT and 35 patients underwent PLT for HCC.

Clinicopathological characteristics, as well as perioperative details, were obtained retrospectively from the computerised clinical and operative databases (Sunrise Clinical Manager version 5.8, Eclipsys Corporation, Atlanta, Georgia, USA and OTM 10, IBM, Armonk, New York, USA). Patient charts were reviewed for supplemental data where necessary. Pre-operative assessments included haematological and biochemical tests (complete blood count, renal and liver function tests, coagulation profile and serum alpha-fetoprotein (AFP)) as well as imaging via computed tomography (CT) and/or magnetic resonance imaging (MRI). Patients who underwent transplantation were assessed at the point of listing for transplantation.

The diagnoses of $\mathrm{HCC}$ and its recurrences were made pre-operatively via imaging, the presence of risk factors and/or serum AFP. Biopsies were performed in selected pre-transplant patients beyond the Milan criteria, for which poor tumour differentiation resulted in their exclusion. Post-operative monitoring included regular liver function tests, test for changes in serum AFP with hepatic ultrasounds and/or CT imaging of the thorax and abdomen.

\section{Definitions}

SLT is referred to liver transplant performed for recurrent HCC and/or deteriorating liver function after pre- vious curative resection. ${ }^{2,9}$ Liver transplant performed for patients who developed recurrence after previous local ablation therapy without prior resection were not considered as SLT. Major liver resection is referred to the resection of three or more liver segments. ${ }^{19}$ Post-operative morbidity and mortality are defined as complications or deaths occurring within 30 days of surgery or within the same hospitalisation of surgery. Morbidity was further classified as major (Grade $\geq 3$ ) and minor (Grade $\leq 2)$ grades according to the Clavien-Dindo classification. ${ }^{20}$ Time to recurrence refers to the point at which tumour recurrence was first identified and documented with clinical and radiological evidence, while recurrence-free survival (RFS) refers to the recording of the time of intervention to disease recurrence or death from any cause.

\section{Statistical analysis}

All statistical analyses were performed using the Statistical Package for Social sciences software for Windows, version 20.0 (SPSS Inc., Chicago, IL). Continuous data are presented as median (range) and analysed via MannWhitney $U$ test while categorical data were analysed via Chi-square test or Fisher's exact test as appropriate. Overall survival (OS) and RFS were calculated by following the Kaplan-Meier method and compared by the logrank test. All survival times were calculated from the time of surgery. Data were censored at the time of the last follow-up for survival analysis. A $p<0.05$ was considered statistically significant.

\section{RESULTS}

Comparison between the two groups demonstrated no significant difference in patient characteristics including age, gender, underlying aetiology and Child-Pugh status. Moreover, there was no significant difference in baseline tumour characteristics such as frequency of tumours within Milan criteria, tumour size, AFP and previous locoregional treatment including radiofrequency ablation (RFA), transarterial chemoembolization (TACE) and Yttrium-90 (Y-90) radioembolization between the groups (Table 1).

The two groups were similar in the pathological features of the explanted liver with regards to frequency of cirrhosis, largest tumour size, number of nodules, tumour differentiation, microvascular invasion and satellite nodules. 
Table 1. Clinicopathologic characteristics of patients undergoing primary and salvage transplant for hepatocellular carcinoma

\begin{tabular}{|c|c|c|c|}
\hline & Primary transplant $(\mathrm{n}=35)$ & Salvage transplant $(n=14)$ & $p$-value \\
\hline Median age, years (range) & $60(46-70)$ & $57(26-69)$ & 0.334 \\
\hline Gender (male) & $30(85.7 \%)$ & $13(92.9 \%)$ & 0.659 \\
\hline \multicolumn{4}{|l|}{ Aetiology } \\
\hline Hepatitis B & $20(57.1 \%)$ & $10(71.4 \%)$ & 0.518 \\
\hline Hepatitis $\mathrm{C}$ & $3(8.6 \%)$ & $1(7.1 \%)$ & 1.000 \\
\hline Non-B/C & $12(34.3 \%)$ & $3(21.4 \%)$ & 0.502 \\
\hline Child-Pugh status & & & 0.441 \\
\hline A & $13(37.1 \%)$ & $8(57.1 \%)$ & \\
\hline $\mathrm{B}$ & $18(51.4 \%)$ & $5(35.7 \%)$ & \\
\hline $\mathrm{C}$ & $4(11.4 \%)$ & $1(7.1 \%)$ & \\
\hline Within Milan criteria & $29(82.9 \%)$ & $11(78.6 \%)$ & 0.702 \\
\hline Median tumour size, cm (range) & $2.3(0-10)$ & $2.45(1-4.6)$ & 0.842 \\
\hline \multicolumn{4}{|l|}{$\mathrm{AFP}, \mathrm{ng} / \mathrm{ml}$} \\
\hline Median (range) & $10.1(2.1-2153)$ & $13.65(2.01-3169)$ & 0.528 \\
\hline$\leq 200$ & $31(88.6 \%)$ & $10(71.4 \%)$ & 0.202 \\
\hline$>200$ & $4(11.4 \%)$ & $4(28.6 \%)$ & \\
\hline Pre-transplant locoregional treatment & $26(74.3 \%)$ & $13(92.9 \%)$ & 0.244 \\
\hline Type of donor transplant & & & 1.000 \\
\hline LDLT & $12(34.3 \%)$ & $4(28.6 \%)$ & \\
\hline DDLT & $23(65.7 \%)$ & $10(71.4 \%)$ & \\
\hline \multicolumn{4}{|l|}{ Pathological characteristics } \\
\hline Liver cirrhosis & $35(100 \%)$ & $14(100 \%)$ & \\
\hline \multicolumn{4}{|l|}{ Largest tumour size } \\
\hline Median, cm (range) & $2.0(0-11.4)$ & $1.1(0-5)$ & 0.356 \\
\hline$\leq 3 \mathrm{~cm}$ & $25(73.5 \%)$ & $11(78.6 \%)$ & 1.000 \\
\hline$>3 \mathrm{~cm}$ & $9(26.5 \%)$ & $3(21.4 \%)$ & \\
\hline \multicolumn{4}{|l|}{ Number of nodules } \\
\hline Median (range) & $1(0-14)$ & $1.5(0-7)$ & 0.610 \\
\hline 1 & $18(51.4 \%)$ & $6(42.9 \%)$ & 0.754 \\
\hline$>1$ & $17(48.6 \%)$ & $8(57.1 \%)$ & \\
\hline Tumour differentiation & & & 0.066 \\
\hline Well & $9(25.7 \%)$ & 0 & \\
\hline Moderate & $9(25.7 \%)$ & $3(21.4 \%)$ & \\
\hline Poor & $8(22.9 \%)$ & $7(50 \%)$ & \\
\hline Complete necrosis & $5(14.3 \%)$ & $4(28.6 \%)$ & \\
\hline Microvascular invasion & $6(17.1 \%)$ & $3(21.4 \%)$ & 0.702 \\
\hline Satellite nodules & $12(34.3 \%)$ & $7(50 \%)$ & 0.346 \\
\hline Median wait time*, months (range) & $8(1-23)$ & $10(3-50)$ & 0.363 \\
\hline
\end{tabular}

AFP, alpha-fetoprotein; RFA, radiofrequency ablation; TACE, transarterial chemoembolization; Y-90, Yttrium-90 microspheres radioembolization; LDLT, living donor liver transplant; DDLT, deceased donor liver transplant

*wait time, time from the listing of transplant to date of transplantation

Also, there was no difference in waiting time calculated from the date of listing to date of transplant.

Postoperative outcomes between both the groups illustrated no significant difference in terms of postoperative morbidity, mortality, intensive care unit (ICU) stay and length of hospital stay (LOS). As shown in Table 2, the SLT group was associated with a non-statistically significant increase in frequency of reoperations $(42.9 \%$ versus $20.0 \%, p=0.152$ ), postoperative renal insufficiency
(35.7\% versus $14.3 \%, p=0.124)$, arterial thrombosis $(14.3 \%$ versus $2.9 \%, p=0.193)$ and bile leak $(14.3 \%$ versus $5.7 \%$, $p=0.568$ ).

Patients were followed up for a median duration of 49.1 months (interquartile range: 13.9-69.5 months). The patients who underwent SLT had a significantly shorter time to recurrence (median=5.5 versus 23 months, $p<0.001$ ) (Table 3 ) and exhibited poorer 5-year OS (PLT $=75 \%$ versus $\mathrm{SLT}=61 \%, p=0.345$, Fig. $1 \mathrm{~A}$ ) and RFS (PLT $=72 \%$ 
Table 2. Postoperative outcomes of patients who underwent primary and salvage transplant

\begin{tabular}{|c|c|c|c|}
\hline & Primary transplant $(n=35)$ & Salvage transplant $(n=14)$ & $p$-value \\
\hline \multicolumn{4}{|l|}{ Postoperative morbidity } \\
\hline Minor morbidity (Grade $\leq 2)$ & $9(25.7 \%)$ & $4(28.6 \%)$ & 1.000 \\
\hline Major morbidity (Grade $\geq 3$ ) & $13(37.1 \%)$ & $6(42.9 \%)$ & 0.711 \\
\hline Reoperation & $7(20 \%)$ & $6(42.9 \%)$ & 0.152 \\
\hline Renal insufficiency & $5(14.3 \%)$ & $5(35.7 \%)$ & 0.124 \\
\hline Sepsis & $5(14.3 \%)$ & $2(14.3 \%)$ & 1.000 \\
\hline Bleeding & $5(14.3 \%)$ & $2(14.3 \%)$ & 1.000 \\
\hline Arterial thrombosis & $1(2.9 \%)$ & $2(14.3 \%)$ & 0.193 \\
\hline Biliary leak & $2(5.7 \%)$ & $2(14.3 \%)$ & 0.568 \\
\hline Acute rejection & $1(2.9 \%)$ & 0 & 1.000 \\
\hline Postoperative mortality & $2(5.7 \%)$ & $1(7.1 \%)$ & 1.000 \\
\hline Median ICU stay, days (range) & $2(2-41)$ & $4.5(1-10)$ & 0.112 \\
\hline Median LOS, days (range) & $19(4-82)$ & $19.5(5-62)$ & 0.973 \\
\hline
\end{tabular}

ICU, intensive care unit; LOS, length of stay

Table 3. Recurrence data of patients who underwent primary and salvage transplant

\begin{tabular}{lccc}
\hline & Primary transplant $(\mathrm{n}=35)$ & Salvage transplant $(\mathrm{n}=14)$ & $p$-value \\
\hline Overall recurrence & $4(11.4 \%)$ & $2(14.3 \%)$ & 1.000 \\
Site of recurrence & & & 0.963 \\
$\quad$ Intrahepatic & $2(5.7 \%)$ & $1(7.1 \%)$ & \\
$\quad$ Intrahepatic \& extrahepatic & $2(5.7 \%)$ & $1(7.1 \%)$ & $<0.001$ \\
Time to recurrence, months & $23(7-59)$ & $5.5(5-6)$ & 0.303 \\
Median OS, months (IQR) & $59(19-71)$ & $33.5(9.5-59.8)$ & 0.228 \\
Median RFS, months (IQR) & $59(9-71)$ & $21(4.3-59.8)$ & \\
\hline
\end{tabular}

RFS, recurrence-free survival; OS, overall survival; IQR, interquartile range
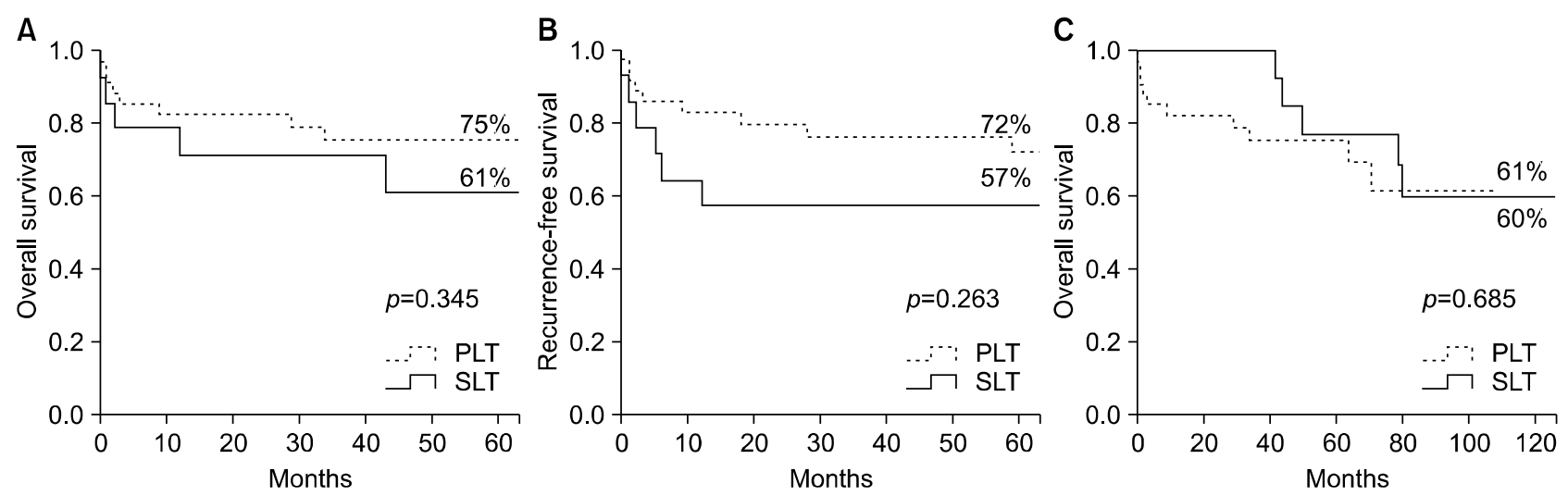

Fig. 1. Comparison of survival outcomes between primary and salvage transplant. (A) Overall survival over 5 years. (B) Recurrence-free survival over 5 years. (C) Overall survival from the point of primary resection over 10 years.

versus $\mathrm{SLT}=57 \%, p=0.263$, Fig. 1B). However, comparisons between SLT patients from the point of primary liver resection and PLT patients trended towards similar OS over a 10 -year period (SLT from primary resection $=60 \%$ versus $\mathrm{PLT}=61 \%, p=0.685$, Fig. $1 \mathrm{C}$ ).

\section{DISCUSSION}

In this study, we investigated the viability of SLT as a treatment strategy for recurrent HCC based on the comparison of survival outcomes and perioperative morbidity 
with that of PLT. Liver transplant is perceived to be the superior treatment modality for HCCs with regards to its improved overall survival and significantly lower cancer recurrence rate. ${ }^{21-23}$ However, the implementation and success are dependent on the availability of specialised transplant expertise and availability of liver grafts.

In the field of transplantation, comparisons between PLT and SLT frequently surfaces with PLT emerging as superior in prolonging life expectancy than SLT with lower perioperative morbidity. ${ }^{6,9}$ SLT was reported to be more technically challenging than PLT, as previous hepatic resection and pre-operative locoregional treatment lead to adhesion formation which hampers tissue dissection and liver mobilisation. The occurrence of more severe adhesions is common in the case of patients who had previous liver resection via the traditional open approach than after laparoscopic liver resection. ${ }^{24,25}$ Dense adhesions result in tedious haemorrhagic dissection, longer operative time and increased blood loss. ${ }^{6}$ Adhesion formation is further compounded by a history of local ablation therapy. In the present series, patients on an average spent a long time on the transplant waiting list than observed in other studies (PLT $=8$ versus $1.9-3$ months and $\mathrm{SLT}=10$ versus $3.1-5$ months $)^{6,17}$ due to low liver donor rates in our country. Longer waiting time results in more pretransplant locoregional bridging interventions with consequent inflammation and adhesion formation, resulting in a more technically difficult transplantation.

As demonstrated in Table 2, 42.9\% of our SLT patients required reoperation compared to $20 \%$ of the PLT group, for which, bleeding was the predominant reason for a repeat operation. The reoperation figures, though statistically insignificant, were in general, higher than those reported in the literature (PLT $=3-13 \%$ and SLT $=0-39 \%$ ). ${ }^{6,17}$ We postulate that the relatively high reoperation rates compared to the reported data in the literature could be explained based on the long wait-list time due to the severe shortage of donor grafts in our country. Consequently, many patients underwent multiple preoperative bridging and downstaging treatments prior to transplant including local ablation and transarterial chemoembolization. These treatments resulted in the frequent formation of dense adhesions which along with the presence of portal hypertension and coagulopathy, led to increased bleeding during and after SLT, likely accounting for the higher reoper- ation rates in this series.

While recurrence patterns were largely similar between PLT and SLT, SLT patients exhibited the pattern significantly earlier at 5.5 months than PLT with 23 months, $p<0.001$ (Table 3), which was likely due to comparatively worse tumour biology in patients undergoing SLT. The SLT cohort in our study had a statistically insignificant higher incidence of tumours with poorer prognostic factors including poorly differentiated tumours, satellite nodules and microvascular invasion; all of which contributed to possibly inferior recurrence-free survival and overall survival in SLT patients. Such an observation raises the questions regarding the use of SLT for recurrent HCCs, especially from deceased donor grafts. The trend towards poorer survival for SLT compared to PLT may result in suboptimal usage of a limited and precious resource. Based on our observations, it is suggested that better patient selection for SLT is required to improve long-term outcomes. Poon et al. had previously suggested an integrated therapeutic strategy for PLT and SLT, whereby SLT would benefit patients with small HCCs and preserved liver function and PLT would be more appropriate for those with oligonodular tumours in a cirrhotic liver. ${ }^{2}$ However, our present study demonstrates no significant difference in 10-year OS between PLT and SLT when survival is measured from the time of primary resection, suggesting that upfront PLT may not confer any survival benefit over resection followed by SLT. Furthermore, our study did not include patients who had a recurrence after initial resection and were deemed unsuitable for resection or salvage transplant. One may argue that such "failures" of the salvage strategy could potentially have benefitted from direct PLT.

Tumour recurrence has been a pertinent issue in the management of HCCs. Underlying liver cirrhosis predisposes to multiple tumours and poses a problem of recurrence, which is unlikely to resolve even after repeated resections. Furthermore, liver cirrhosis and reduced remnant liver volume poses the likelihood of post-hepatectomy liver failure and limits the feasibility of repeated liver resections. ${ }^{17}$ There exists a need to eliminate the underlying diseased liver that results in inevitable tumour recurrence and compromises future survival.

While locoregional therapy bridges patients over to eventual transplant, they are by no means infallible and 
patients run the risk of tumour recurrence while on the waitlist. The use of living donor grafts for liver transplant may circumvent this issue and shorten waiting time with a potential gain in survival and cost-effectiveness. ${ }^{26}$ In the absence of a suitable liver graft, however, RLR is a potentially safe and viable option as it offers a bridge to eventual SLT. ${ }^{27}$ Though secondary to transplant in terms of disease-free survival benefit, its outcomes may be better improved with the advent of locoregional and systemic therapies. Laparoscopic primary liver resection has been demonstrated to have fewer perioperative complications with reduced LOS while open resection gives rise to increased adhesions, heightening intraoperative dissection efforts with prolonged operative time ${ }^{24,25,28}$ The benefits of laparoscopy may potentially extend to resections for recurrent tumours, which has been postulated to be a safe and feasible operation with laparoscopic redo surgery made easier in patients with previous laparoscopic resections compared to open resections. ${ }^{29,30}$ The utility of laparoscopic re-resections for tumour recurrences may be further explored as the need for hepatic re-resection persists in the midst of liver graft shortages. Advanced planning of conglomeration of surgical and patient optimisation for the treatment of HCC may be essential for eventual preparation for inevitable transplant and translation into reduced perioperative morbidity and increased survival benefit.

Although the data from the present retrospective study is largely congruent to previous papers, it is limited due to a small sample size that prevents the study from being adequately powered in detecting true statistical significance. Selection bias may be inherent in the matching process as part of a non-randomized retrospective study and incorporating patients beyond the Milan criteria together with the simultaneous application of multiple treatment modalities (i.e., locoregional treatment) may confound overall analysis. Nevertheless, these results may be useful in guiding clinicians and patients for or against SLT. Patients should be advised about the risks and benefits of each therapeutic strategy in making an informed decision with respect to the availability of liver grafts. Even as transplant shows benefit in RFS and should be offered as a first-line treatment for a selective group of patients, we are limited by scarce liver grafts which emphasises the need for risk stratification and resource allocation so that their utility reaps the most benefit.

In conclusion, SLT is a viable treatment option for the management of HCCs. However, it exhibited poorer shortterm perioperative and long-term oncologic outcomes compared to PLT. Better patient selection is needed for SLT especially with regards to deceased donor grafts in order to optimize resource allocation in this era of organ shortage.

\section{REFERENCES}

1. Ferlay J, Soerjomataram I, Dikshit R, Eser S, Mathers C, Rebelo $\mathrm{M}$, et al. Cancer incidence and mortality worldwide: sources, methods and major patterns in GLOBOCAN 2012. Int J Cancer 2015;136:E359-E386.

2. Poon RT, Fan ST, Lo CM, Liu CL, Wong J. Long-term survival and pattern of recurrence after resection of small hepatocellular carcinoma in patients with preserved liver function: implications for a strategy of salvage transplantation. Ann Surg 2002;235: 373-382.

3. Llovet JM, Fuster J, Bruix J. Intention-to-treat analysis of surgical treatment for early hepatocellular carcinoma: resection versus transplantation. Hepatology 1999;30:1434-1440.

4. Figueras J, Jaurrieta E, Valls C, Benasco C, Rafecas A, Xiol X, et al. Survival after liver transplantation in cirrhotic patients with and without hepatocellular carcinoma: a comparative study. Hepatology 1997;25:1485-1489.

5. Bhangui P, Allard MA, Vibert E, Cherqui D, Pelletier G, Cunha AS, et al. Salvage versus primary liver transplantation for early hepatocellular carcinoma: do both strategies yield similar outcomes? Ann Surg 2016;264:155-163.

6. Adam R, Azoulay D, Castaing D, Eshkenazy R, Pascal G, Hashizume $\mathrm{K}$, et al. Liver resection as a bridge to transplantation for hepatocellular carcinoma on cirrhosis: a reasonable strategy? Ann Surg 2003;238:508-518; discussion 518-519.

7. Hu Z, Wang W, Li Z, Ye S, Zheng SS. Recipient outcomes of salvage liver transplantation versus primary liver transplantation: a systematic review and meta-analysis. Liver Transpl 2012;18: 1316-1323.

8. Ng KK, Lo CM, Liu CL, Poon RT, Chan SC, Fan ST. Survival analysis of patients with transplantable recurrent hepatocellular carcinoma: implications for salvage liver transplant. Arch Surg 2008;143:68-74; discussion 74.

9. Majno PE, Sarasin FP, Mentha G, Hadengue A. Primary liver resection and salvage transplantation or primary liver transplantation in patients with single, small hepatocellular carcinoma and preserved liver function: an outcome-oriented decision analysis. Hepatology 2000;31:899-906.

10. Poon RT, Fan ST, Lo CM, Liu CL, Wong J. Intrahepatic recurrence after curative resection of hepatocellular carcinoma: long-term results of treatment and prognostic factors. Ann Surg 1999;229:216-222.

11. Tung-Ping Poon R, Fan ST, Wong J. Risk factors, prevention, and management of postoperative recurrence after resection of hepatocellular carcinoma. Ann Surg 2000;232:10-24.

12. Shimada M, Takenaka K, Taguchi K, Fujiwara Y, Gion T, Kajiyama K, et al. Prognostic factors after repeat hepatectomy for recurrent hepatocellular carcinoma. Ann Surg 1998;227:80-85.

13. Lee PH, Lin WJ, Tsang YM, Hu RH, Sheu JC, Lai MY, et al. 
Clinical management of recurrent hepatocellular carcinoma. Ann Surg 1995;222:670-676.

14. Faber W, Seehofer D, Neuhaus P, Stockmann M, Denecke T, Kalmuk S, et al. Repeated liver resection for recurrent hepatocellular carcinoma. J Gastroenterol Hepatol 2011;26:1189-1194.

15. Huang ZY, Liang BY, Xiong M, Zhan DQ, Wei S, Wang GP, et al. Long-term outcomes of repeat hepatic resection in patients with recurrent hepatocellular carcinoma and analysis of recurrent types and their prognosis: a single-center experience in China. Ann Surg Oncol 2012;19:2515-2525.

16. Fuks D, Dokmak S, Paradis V, Diouf M, Durand F, Belghiti J. Benefit of initial resection of hepatocellular carcinoma followed by transplantation in case of recurrence: an intention-to-treat analysis. Hepatology 2012;55:132-140.

17. Belghiti J, Cortes A, Abdalla EK, Régimbeau JM, Prakash K, Durand F, et al. Resection prior to liver transplantation for hepatocellular carcinoma. Ann Surg 2003;238:885-892; discussion 892-3.

18. Del Gaudio M, Ercolani G, Ravaioli M, Cescon M, Lauro A, Vivarelli $\mathrm{M}$, et al. Liver transplantation for recurrent hepatocellular carcinoma on cirrhosis after liver resection: University of Bologna experience. Am J Transplant 2008;8:1177-1185.

19. Bismuth $\mathrm{H}$. Surgical anatomy and anatomical surgery of the liver. World J Surg 1982;6:3-9.

20. Dindo D, Demartines N, Clavien PA. Classification of surgical complications: a new proposal with evaluation in a cohort of 6336 patients and results of a survey. Ann Surg 2004;240:205-213.

21. Bismuth H, Chiche L, Adam R, Castaing D, Diamond T, Dennison A. Liver resection versus transplantation for hepatocellular carcinoma in cirrhotic patients. Ann Surg 1993;218: 145-151.

22. Bigourdan JM, Jaeck D, Meyer N, Meyer C, Oussoultzoglou E, Bachellier P, et al. Small hepatocellular carcinoma in Child A cirrhotic patients: hepatic resection versus transplantation. Liver Transpl 2003;9:513-520.

23. Lee KK, Kim DG, Moon IS, Lee MD, Park JH. Liver transplantation versus liver resection for the treatment of hepatocellular carcinoma. J Surg Oncol 2010;101:47-53.

24. Belli G, Fantini C, D'Agostino A, Cioffi L, Langella S, Russolillo N, et al. Laparoscopic versus open liver resection for hepatocellular carcinoma in patients with histologically proven cirrhosis: short- and middle-term results. Surg Endosc 2007;21: 2004-2011.

25. Cheung TT, Poon RT, Yuen WK, Chok KS, Jenkins CR, Chan $\mathrm{SC}$, et al. Long-term survival analysis of pure laparoscopic versus open hepatectomy for hepatocellular carcinoma in patients with cirrhosis: a single-center experience. Ann Surg 2013;257: 506-511.

26. Sarasin FP, Majno PE, Llovet JM, Bruix J, Mentha G, Hadengue A. Living donor liver transplantation for early hepatocellular carcinoma: a life-expectancy and cost-effectiveness perspective. Hepatology 2001;33:1073-1079.

27. Itamoto $\mathrm{T}$, Nakahara $\mathrm{H}$, Amano $\mathrm{H}$, Kohashi $\mathrm{T}$, Ohdan $\mathrm{H}$, Tashiro $\mathrm{H}$, et al. Repeat hepatectomy for recurrent hepatocellular carcinoma. Surgery 2007;141:589-597.

28. Reddy SK, Tsung A, Geller DA. Laparoscopic liver resection. World J Surg 2011;35:1478-1486.

29. Belli G, Cioffi L, Fantini C, D'Agostino A, Russo G, Limongelli $\mathrm{P}$, et al. Laparoscopic redo surgery for recurrent hepatocellular carcinoma in cirrhotic patients: feasibility, safety, and results. Surg Endosc 2009;23:1807-1811.

30. Goh BK, Teo JY, Chan CY, Lee SY, Cheow PC, Chung AY. Review of 103 cases of laparoscopic repeat liver resection for recurrent hepatocellular carcinoma. J Laparoendosc Adv Surg Tech A 2016;26:876-881. 